\title{
RANDOM SYSTEMS IN ULTRAMETRIC SPACES
}

\author{
BY D. A. DAWSON AND L. G. GOROSTIZA
}

\begin{abstract}
We discuss percolation and random walks in a class of homogeneous ultrametric spaces together with similarities and differences in ultrametric and Euclidean spaces. We briefly outline the role of these models in the study of interacting systems. Several open problems are presented.

Keywords: Ultrametric space; hierarchical lattice; random graph; percolation; random walk; interacting system; branching

2010 Mathematics Subject Classification: Primary 05C80; 05C81; 60K35

Secondary $60 \mathrm{C} 05$
\end{abstract}

\section{Introduction}

An ultrametric space is given by a pair $(S, d)$, where $S$ is a set and $d$ is a metric, called an ultrametric, that satisfies the strong (non-Archimedean) triangle inequality

$$
d(x, y) \leq \max \{d(x, z), d(z, y)\} \quad \text { for any } x, y, z \in S .
$$

Basic properties of ultrametric spaces can be found in [35]. The $p$-adic numbers $\mathbb{Q}_{p}$ constitute a field with an ultrametric which plays an important role in algebra and number theory, and $p$-adic mathematical analysis exists. $\mathbb{Q}_{p}$ and other ultrametric spaces are often used in mathematical physics [7], [18]. Ultrametric structures of different types occur in many areas, including statistical physics and biology [34], in particular genealogical trees [21], the Parisi ultrametricity conjecture for spin glass [33], and the dynamics of protein molecules [1].

In this paper we present an overview of results on percolation and the so-called hierarchical random walk on a class of ultrametric spaces known as hierarchical lattices. We also discuss a nearest-neighbour random walk on percolation clusters, and mention some hierarchically organized interacting systems, in particular branching systems. We show how some results in [12] can be extended to a larger class of hierarchical lattices, and present some open problems. There are similarities and differences for analogous random systems on ultrametric and Euclidean spaces. Some things are simpler on hierarchical lattices due to the ultrametric structure (e.g. renormalization methods of statistical physics; these have been used in [10], [11], and [12]).

\section{A class of homogeneous ultrametric lattices}

\subsection{Definition and metric structure}

We introduce a class of ultrametric lattices analogous to the homogeneous Euclidean lattices such as $\mathbb{Z}^{d}$. A homogeneous ultrametric lattice $\Omega$ is defined by

(i) a countable set $\Omega$ furnished with an ultrametric $d$ such that, for any $\omega \in \Omega$ and $r \in(0, \infty)$, the $r$-ball (ball of diameter $r$ ) containing $\omega \in \Omega$ has finitely many elements; and 
(ii) given any two elements $\omega_{1}, \omega_{2} \in \Omega$, there exists a bijective mapping $\rho: \Omega \rightarrow \Omega$ such that $\rho\left(\omega_{1}\right)=\omega_{2}$ and $\left.d\left(\rho(\omega), \rho\left(\omega^{\prime}\right)\right)=d\left(\omega, \omega^{\prime}\right)\right)$ for all $\omega, \omega^{\prime} \in \Omega$.

Since an increasing function of an ultrametric is an ultrametric, without loss of generality we can assume that the ultrametric is integer valued and that there is a nested sequence of balls with diameters $1,2,3, \ldots$ Given any sequence $N_{i} \in \mathbb{N} \backslash\{1\}, i=1,2, \ldots$, define $\Omega_{N_{1}, N_{2}, \ldots} \equiv \Omega_{\left\{N_{i}\right\}}$ to be the homogeneous ultrametric lattice in which a $k$-ball contains $N_{k}(k-1)$-balls (a 0 -ball is identified with an element of $\Omega$ ). Define $\hat{N}_{k}=\prod_{i=1}^{k} N_{i}$; this is the size of a $k$-ball. Then each point has $\hat{N}_{k}-\hat{N}_{k-1}$ points at distance $k$.

\subsection{Algebraic structure and tree representation}

The ultrametric lattice $\Omega_{\left\{N_{i}\right\}}$ has an Abelian group structure whose elements are sequences, defined by the direct sum

$$
\Omega_{\left\{N_{i}\right\}}=\bigoplus_{i=1}^{\infty} \mathbb{Z}_{N_{i}}=\left\{x=\left(x_{1}, x_{2}, \ldots\right), x_{i} \in \mathbb{Z}_{N_{i}} \text { for all } i, \sum_{i} x_{i}<\infty\right\},
$$

where $\mathbb{Z}_{N_{i}}=\left\{0,1, \ldots, N_{i}-1\right\}$ is the cyclic group of order $N_{i}$, with addition componentwise $\left(\bmod N_{i}\right)$ for each $i$. We represent $\Omega_{\left\{N_{i}\right\}}$ as the leaves at the top of an infinite tree, where each node at level $k$ from the top (which is level 0 ) is connected by branches to $N_{k}$ nodes at level $k-1$ and to one node at level $k+1$. The distance between two points $x$ and $y$ defined by $d(x, y)=\max \left\{k: x_{k} \neq y_{k}\right\}$ satisfies the strong triangle inequality. We call $\Omega_{\left\{N_{i}\right\}}$ a hierarchical lattice (or hierarchical group) because of the hierarchy induced by the distance, and $d(x, y)$ is called the hierarchical distance. Balls in $\Omega_{\left\{N_{i}\right\}}$ can be represented by inner nodes of the tree. (Due to componentwise addition, if $N_{i}=p$ prime for all $i$, in contrast to $\mathbb{Q}_{p}$, there is no carry in the addition operation; however, $d(0, x+y) \leq \max \{d(0, x), d(0, y)\}$ in both cases.) The distance between $x$ and $y$ is represented on the tree by the level of the node corresponding to the smallest ball containing $x$ and $y$. The point $0=(0,0, \ldots)$ can be taken as the origin and as a spine of the tree. In Figure 1 we illustrate $\Omega_{\{3,2,2, \ldots\}}$ up to hierarchical distance 3 .

In contrast to Euclidean lattices, in a hierarchical lattice it is not possible to go arbitrarily far with a sequence of steps of uniformly bounded sizes, neither can there be an infinite connected subset with connections between nearest neighbours. For this reason, random walks on Euclidean lattices and on $\Omega_{\left\{N_{i}\right\}}$ are quite different, and percolation in $\Omega_{\left\{N_{i}\right\}}$ can exist only as long-range percolation.

When $N_{i}=N$ for all $i$, we denote $\Omega_{\left\{N_{i}\right\}}$ by $\Omega_{N}$. In this case, in spite of the different geometric properties of $\mathbb{Z}^{d}$ and $\Omega_{N}$, there is a special random walk on $\Omega_{N}$ that behaves

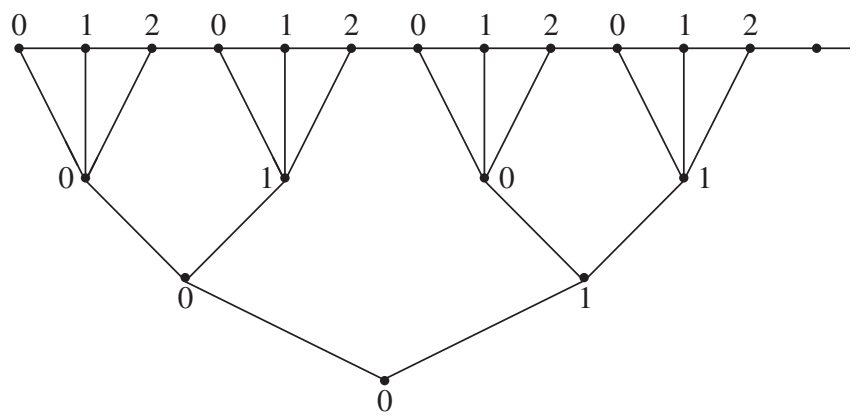

FIGURE 1. 
analogously to the simple random walk on $\mathbb{Z}^{d}$. This is because for random walks on both $\mathbb{Z}^{d}$ and $\Omega_{N}$, the long-time behaviour and other properties depend only on the degree of transience and recurrence (we show this in Subsection 4.2). There is also a continuum hierarchical group where Lévy processes can be defined [17]. This is referred to in Subsection 5.1.

There are hierarchical lattices that are ultrametric but are not groups, e.g. if two nodes at level $k$ are connected by branches to different numbers of nodes at level $k-1$.

\section{Percolation in homogeneous ultrametric lattices}

\subsection{The family of ultrametric random graphs}

Fix $\left\{N_{i}\right\}$ and a function $q: \mathbb{N} \rightarrow(0,1)$. We consider the family of ultrametric random graphs $\mathcal{G}_{\left\{N_{i}\right\}}^{\infty}=(V, \mathcal{E})$ with vertices $V=\Omega_{\left\{N_{i}\right\}}$, and a random set of (undirected) edges $\mathscr{E} \subset \Omega_{\left\{N_{i}\right\}} \times \Omega_{\left\{N_{i}\right\}}$ such that

(i) the events $\{(x, y) \in \mathcal{E}\}$ are independent; and

(ii) $\mathbb{P}((x, y) \in \mathcal{E})=q(d(x, y))$, where $d(x, y)$ is the hierarchical distance on $\Omega_{\left\{N_{i}\right\}}$.

Thus, $q(k)$ is the probability of connection between two points separated by distance $k ; q(k)$ plays a basic role in percolation.

A collection of vertices in a subset of $\Omega_{\left\{N_{i}\right\}}$, any two of which are linked by a path of edges, is called a cluster of the subset. For each $k$-ball, $k \geq 1$, a maximal cluster is defined as having all its edges within the ball (i.e. it has no paths that go outside the ball, so all edges of length less than or equal to $k$ not in the cluster are deleted). If there exist two or more maximal clusters then choose one of them uniformly at random. In this way each $k$-ball has a unique attached cluster. Say that percolation in $\Omega_{\left\{N_{i}\right\}}$ occurs if the probability that a designated vertex 0 (e.g. $0=(0,0, \ldots))$ belongs to an infinite cluster, called a percolation cluster, is positive. Consider the cluster containing 0 . Ignoring collisions, we can compare this to a Galton-Watson branching process in which the offspring of a vertex are the elements of $\Omega_{\left\{N_{i}\right\}}$ connected to it by single edges. The expected number of offspring of a vertex is

$$
m=\sum_{k=1}^{\infty} q(k)\left(\hat{N}_{k}-\hat{N}_{k-1}\right) ;
$$

hence, if $m<1$ then the branching process is subcritical and, therefore, the cluster is finite with probability 1 , so percolation does not occur. The question that now arises is that of the conditions on the connection probabilities $q(k)$ that lead to a percolation cluster.

We now describe in the context of $\Omega_{\left\{N_{i}\right\}}$ the approach developed in the series of papers [10], [11], and [12] for $\Omega_{N}$ to establish percolation or non-percolation (see also [25] and [36] for other approaches and a special type of connection probability). This is based on identifying an associated hierarchy of random Erdős-Rényi graphs. A random Erdős-Rényi graph $q=g(V, \mathcal{E})$ is a random graph with a finite set of vertices $V$, independent and identically distributed (i.i.d.) random weights $\left\{w_{i} \in[0,1]: i \in V\right\}$, and $\mathbb{P}((i, j) \in \mathcal{E})=p\left(w_{i}, w_{j}\right)$, a function of $w_{i}$ and $w_{j}$, with the events $(i, j) \in \mathcal{E}$ being independent conditioned on the weights.

We construct the hierarchy of random Erdős-Rényi graphs

$$
g_{k}=g\left(V_{k},\left\{X_{k-1}(i)\right\}_{i \in V_{k}}\right), \quad k \geq 1,
$$

as follows. Let $\hat{N}_{j}^{k}=\hat{N}_{k} / \hat{N}_{j}$ denote the number of (disjoint) $j$-balls in a $k$-ball, $j<k$. At the $k$ th level the vertices are given by the $\hat{N}_{k-1}^{k}(k-1)$-balls in the $k$-ball $B_{k}(0)$ containing the 
designated vertex (note that $\hat{N}_{k-1}^{k}=N_{k}$ in this case). The weights $\left\{X_{k-1}(i)\right\}$ are defined as follows: $X_{0}(i)=1$ and, for $k \geq 2$, the $\left\{X_{k-1}(i)\right\}_{i \in V_{k}}$ are the densities of the $\hat{N}_{k-1}^{k}(k-1)$-balls in $B_{k}(0)$ indexed by $V_{k},\left|V_{k}\right|=\hat{N}_{k-1}^{k}$. The densities $\left\{X_{k-1}(i)\right\}_{i \in V_{k}}$ are i.i.d. [0,1]-valued random variables. For each $k$,

$$
X_{k-1}(i)=\frac{\left|\mathcal{C}_{k-1}(i)\right|}{\hat{N}_{k-1}}, \quad i \in V_{k},
$$

where $\mathcal{C}_{k-1}(i)$ denotes the cluster in the $i$ th $(k-1)$-ball. For $i \neq j \in V_{k}$, the (random) probability that the $(k-1)$-balls indexed $i$ and $j$ are connected through their clusters by an edge is

$$
p_{k}(i, j)=1-[1-q(k)]^{\left(\hat{N}_{k-1}\right)^{2} X_{k-1}(i) \cdot X_{k-1}(j)} .
$$

Our aim now is to use this hierarchy to obtain criteria for percolation in $\mathcal{G}_{\left\{N_{i}\right\}}^{\infty}$.

Denote the distribution of $X_{k}(i)$ by $\mu_{k} \in \mathcal{P}([0,1])$. Since the density of the $k$-ball $B_{k}(0)$ is given by

$$
X_{k}=\frac{1}{N_{k}} \sum_{i} X_{k-1}(i),
$$

where the sum runs over the set of $(k-1)$-balls in $B_{k}(0)$ that are all connected through their clusters and the $X_{k-1}(i)$ are i.i.d., then, for each $k, \mu_{k}$ is a function of $\mu_{k-1}$ which plays the role of a renormalization mapping.

We analyse the sequence $\mu_{k}, k \geq 1$, for the class of connection probabilities given below. In order to prove percolation of positive density, it suffices to construct a sequence of $[0,1]$-valued random variables $X_{k}, k \geq 1$, satisfying

$$
\mathbb{P}\left(X_{k}>a\right)=\mu_{k}((a, 1]) \quad \text { for all } a \in(0,1),
$$

for some $a>0$ such that

$$
\liminf _{k \rightarrow \infty} \mathbb{P}\left(X_{k}>a\right)>0
$$

\subsection{Percolation in $\boldsymbol{\Omega}_{\left\{N_{i}\right\}}$}

Suppose that the connection probabilities are expressible as

$$
q(k)=\min \left(\frac{c_{k}}{\hat{N}_{k}^{(1+\delta)}}, 1\right),
$$

where $\delta>0$ and the $\left\{c_{k}\right\}$ satisfy $0<\inf c_{k} \leq \sup c_{k}<\infty$. We adapt the method used in [11] for $\Omega_{N}$ to prove existence of percolation clusters in the ultrametric lattice $\Omega_{\left\{N_{i}\right\}}$ when $\left\{N_{i}\right\}$ is bounded.

Theorem 1. (a) If $N_{i} \geq 2$ for all $i$ and $\delta>1$ then percolation does not occur.

(b) Assume that $2 \leq N_{i} \leq M$ for all $i$ and $\delta<1$. If $c=\inf c_{k}$ is sufficiently large then 0 belongs to an infinite cluster with positive probability.

Proof. (a) If $N_{i} \geq 2$ for all $i$ and $\delta>1$ then a simple calculation shows that

$\mathbb{P}\left(B_{k}(0)\right.$ is connected to its complement for infinitely many $\left.k\right)=0$,

so there is no percolation.

(b) We sketch the argument. Let $k_{n}=\lfloor n \log n\rfloor$, so that $k_{n+1}-k_{n} \sim \log n$. Choose $\gamma$ such that $\frac{1}{2}(1+\delta)<\gamma<1$. 
We use an hierarchy of random Erdős-Rényi graphs

$$
g_{n+1}=g\left(\hat{N}_{k_{n}}^{k_{n+1}},\left\{X_{n}(i), i=1, \ldots, \hat{N}_{k_{n}}^{k_{n+1}}\right\}\right),
$$

where $\hat{N}_{k_{n}}^{k_{n+1}}$ is the number of $k_{n}$-balls in a $k_{n+1}$-ball. The weights $\left\{X_{n}(i), i=1, \ldots, \hat{N}_{k_{n}}^{k_{n+1}}\right\}$ are the densities of the $\hat{N}_{k_{n}}^{k_{n+1}} k_{n}$-balls in the $k_{n+1}$-ball $B_{k_{n+1}}(0)$. For $\gamma$ chosen as above, say that the $k_{n}$-ball indexed by $i$ is $\gamma$-good if the cluster it contains, $\mathcal{C}_{k_{n}}(i)$, satisfies $\left|\mathcal{C}_{k_{n}}(i)\right| \geq \hat{N}_{k_{n}}^{\gamma}$. Let $p_{n}$ denote the conditional probability that $B_{k_{n}}(0)$ is $\gamma$-good given that $B_{k_{n-1}}(0)$ is $\gamma$-good, i.e.

$$
p_{n}=\mathbb{P}\left(\left|\mathcal{C}_{k_{n}}(0)\right| \geq \hat{N}_{k_{n}}^{\gamma}|| \mathcal{C}_{k_{n-1}}(0) \mid \geq \hat{N}_{k_{n-1}}^{\gamma}\right) .
$$

Then the renormalization mapping $\mu_{k_{n}} \rightarrow \mu_{k_{n+1}}$ that ensues from the one in the previous subsection implies an induced renormalization mapping $p_{n} \rightarrow p_{n+1}$.

Choose $n_{0}$ large enough so that

$$
\frac{1}{2} \hat{N}_{k_{n_{0}}}^{k_{n_{0}+1}} \gg\left(\hat{N}_{k_{n_{0}}}^{k_{n_{0}+1}}\right)^{\gamma}
$$

and assume that

$$
p_{n_{0}}>\frac{1}{2}
$$

(we verify this assumption later in this proof). Then the probability of connection between two clusters $\mathcal{C}_{k_{n}}(i)$ and $\mathcal{C}_{k_{n}}(j)$ in different $\gamma$-good $k_{n}$-balls in a $k_{n+1}$-ball is bounded below by

$$
\tilde{q}_{n}=1-\left(1-\frac{c}{\hat{N}_{k_{n+1}}^{1+\delta}}\right)^{\left|\mathcal{C}_{k_{n}}(i) \| \mathcal{C}_{k_{n}}(j)\right|} \geq 1-\left(1-\frac{c}{\hat{N}_{k_{n+1}}^{1+\delta}}\right)^{\hat{N}_{k_{n}}^{2 \gamma}} \geq 1-\exp \left(-\frac{c \hat{N}_{k_{n}}^{2 \gamma}}{\hat{N}_{k_{n+1}}^{1+\delta}}\right) .
$$

Let $Y_{n}$ denote the number of $\gamma$-good $k_{n}$-balls in the $k_{n+1}$-ball $B_{k_{n+1}}(0)$. If $Y_{n} \geq\left(\hat{N}_{k_{n}}^{k_{n+1}}\right)^{\gamma}$ and if at least a subset of $\left(\hat{N}_{k_{n}}^{k_{n+1}}-1\right)^{\gamma}$ of the $Y_{n} \gamma$-good $k_{n}$-balls are connected to $B_{k_{n}}(0)$, assumed to be $\gamma$-good, then the cluster in $B_{k_{n+1}}(0)$ has size greater than or equal to $\left(\hat{N}_{k_{n}}^{k_{n+1}}\right)^{\gamma}$, and so $B_{k_{n+1}}(0)$ is $\gamma$-good. Therefore,

where

$$
p_{n+1} \geq \mathbb{P}\left(Y_{n} \geq\left(\hat{N}_{k_{n}}^{k_{n+1}}\right)^{\gamma}\right) \tilde{q}_{n}^{\left(\hat{N}_{k_{n}}^{k_{n+1}}\right)^{\gamma}} \geq \mathbb{P}\left(Y_{n} \geq\left(\hat{N}_{k_{n}}^{k_{n+1}}\right)^{\gamma}\right)\left(1-\varepsilon_{n}\right),
$$

$$
\begin{aligned}
\varepsilon_{n} & =1-\left(1-\exp \left(-\frac{c \hat{N}_{k_{n}}^{2 \gamma-(1+\delta)}}{\left(\hat{N}_{k_{n+1}}^{k_{n+1}}\right)^{1+\delta}}\right)\right)^{\left(\hat{N}_{k_{n}}^{k_{n+1}}\right)^{\gamma}} \\
& \lesssim M^{\gamma \log n} \exp \left\{-c 2^{\sigma n \log n} M^{-(1+\delta) \log n}\right\}
\end{aligned}
$$

and $\sigma=2 \gamma-(1+\delta)>0$.

Now $Y_{n}$ is $\operatorname{Bin}\left(\hat{N}_{k_{n}}^{k_{n}+1}, p_{n}\right)$, and by a standard large deviation estimate [24, Equation (2.6), p. 26], we have

$$
\mathbb{P}\left(Y_{n_{0}}>\left(\hat{N}_{k_{n_{0}}}^{k_{n_{0}+1}}\right)^{\gamma}\right) \geq 1-\exp \left(-\frac{\left[\hat{N}_{k_{n_{0}}}^{k_{n_{0}+1}} p_{n_{0}}-\left(\hat{N}_{k_{n_{0}}}^{k_{n_{0}+1}}\right)^{\gamma}\right]^{2}}{2 \hat{N}_{k_{n_{0}}}^{k_{n_{0}+1}} p_{n_{0}}}\right) \geq 1-\mathrm{e}^{c_{*} n_{0}^{\log 2}}
$$

for some $c_{*}>0$, since we have assumed that $N_{i} \geq 2$ and $p_{n_{0}} \geq \frac{1}{2}$. Then

$$
p_{n_{0}+1} \geq\left(1-\mathrm{e}^{-c_{*} n_{0}^{\log 2}}\right)\left(1-M^{\gamma \log n_{0}} \exp \left(-c 2^{\sigma n_{0} \log n_{0}} M^{-(1+\delta) \log n_{0}}\right)\right) .
$$


Now choose $n_{0}$ so that the right-hand side is greater than $\frac{1}{2}$. Then, by choosing the $c_{i}$ so that $\inf _{i \leq n_{0}} c_{i}$ is sufficiently large, we can satisfy $p_{n_{0}}>\frac{1}{2}$ (see [11, Section 4] for details). Then $p_{n_{0}+2}$ is at least as large as

$$
\left(1-\mathrm{e}^{-c_{*}\left(n_{0}+1\right)^{\log 2}}\right)\left(1-M^{\gamma \log \left(n_{0}+1\right)} \exp \left(-c 2^{\sigma\left(n_{0}+1\right) \log \left(n_{0}+1\right)} M^{-(1+\delta) \log \left(n_{0}+1\right)}\right)\right) .
$$

Furthermore, for every $n$ for which $p_{n}>\frac{1}{2}$, we can iterate the steps above so as to obtain a sequence $\left\{p_{n}\right\}$ satisfying

$$
\sum_{k=n_{0}}^{\infty}\left(1-p_{k}\right)<\infty
$$

and, therefore, $\prod_{k=n_{0}}^{\infty} p_{k}>0$. Thus, there is positive probability of a nested sequence of $\gamma$-good $k_{n}$-balls with nested clusters $\mathcal{C}_{k_{n}}$, which proves percolation.

The critical case $\delta=1$ is more subtle; for this case, we consider $N_{i} \equiv N$ and regard the model as the infinite random graph

$$
\mathcal{g}_{N}^{\infty}=\mathcal{g}_{N}^{\infty}\left(C_{0}, C_{1}, C_{2}, \alpha\right):=g_{(}(V, \mathcal{E})
$$

with vertices $V=\Omega_{N}$ and edges $\mathcal{E}$, and with connection probabilities

$$
q(k)=\min \left(\frac{c_{k}}{N^{2 k}}, 1\right)
$$

where the $c_{k}$ are of the form

$$
c_{k}=C_{0}+C_{1} \log k+C_{2} k^{\alpha}
$$

with constants $C_{0}, C_{1} \geq 0, C_{2}>0$, and $\alpha>0$. For simplicity, we can set $C_{0}=C_{1}=0$ without loss of generality. The graph $g_{N}^{\infty}$ is a limit of finite graphs of diameter $k$ whose behaviour as $k \rightarrow \infty$ is used to show that $g_{N}^{\infty}$ has an infinite cluster.

Theorem 2. ([12].) Let $\delta=1$. For sufficiently large $C_{2}$, there exists a unique percolation cluster of positive density at least $\varepsilon=\varepsilon\left(N, C_{2}, \alpha\right)$ in $\Omega_{N}$ and to which 0 belongs with positive probability.

Proof. (Strategy of proof.) The proof is again based on the hierarchy of random Erdős-Rényi graphs $\mathcal{G}\left(N,\left\{X_{k-1}(i)\right\}_{i \in V_{k}}\right), k \geq 1$, defined above, in the $N_{i} \equiv N$ case, where $X_{0}(i)=1$ and $\left\{X_{k-1}(i)\right\}_{i \in V_{k}}$ are the densities of the $N(k-1)$-balls in $B_{k}(0)$. To carry out the proof as described at the end of Subsection 3.1, the main step is to show that, as $n \rightarrow \infty$,

$$
\mathbb{E}\left[X_{n}\right] \rightarrow a>0 \quad \text { and } \quad \operatorname{var}\left[X_{n}\right] \rightarrow 0
$$

The calculations start with lower bounds for the probability $\tilde{q}_{n}\left(x_{1}, x_{2}\right)$ that two $(n-1)$-balls in an $n$-ball with densities greater than or equal to $x_{1}$ and $x_{2}$ are not connected through their clusters:

$$
\tilde{q}_{n}\left(x_{1}, x_{2}\right) \geq\left(1-\frac{C_{2} n^{\alpha}}{N^{2 n}}\right)^{N^{2(n-1)} x_{1} x_{2}} ;
$$

denote

$$
q_{n}(\varepsilon)=\tilde{q}_{n}(\varepsilon, \varepsilon), \quad \varepsilon>0 .
$$


The probability $P(N, q)$ that the Erdős-Rényi graph $g(N, 1-q)$ is connected satisfies

$$
P(N, q) \geq 1-C(N) q^{N-1} \quad \text { as } q \rightarrow 0 .
$$

In order for $\mathbb{E}\left[X_{n}\right] \rightarrow a>0$, we verify that $q_{n}\left(X_{n-1}\right) \rightarrow 0$, which is used to show that the $n$th Erdős-Rényi graph is connected for sufficiently large $n$. A subtle point is to handle the balls with small density; these occur with probability

$$
z_{n}(\varepsilon)=\mathbb{P}\left(X_{n}<\varepsilon\right) .
$$

A recursive calculation involving $q_{n}(\cdot)$ and $z_{n}(\cdot)$ is used to prove that there exists $\varepsilon>0$ such that

$$
\mathbb{E}\left[X_{n}\right] \geq \mathbb{E}\left[X_{n+1}\right] \geq \mathbb{E}\left[X_{n}\right]\left(1-\frac{1}{2} q_{n}(\varepsilon)\right)
$$

for all $n$, etc., which gives part of the result. We study $\operatorname{var}\left[X_{n}\right]$ similarly (see [12, Section 2.1] for details).

\subsection{Necessary and sufficient conditions in the mean-field limit}

Additional insight in the $N_{i} \equiv N$ case can be obtained by investigating the mean-field limit, $N \rightarrow \infty$, considering first the finite hierarchies of level $k$ in the limit $N \rightarrow \infty$ and then taking $k \rightarrow \infty$.

Assume that the connection probability at distance $k$ is $q(k)=c_{k} / N^{2 k-1}$. Mean-field percolation is said to occur if

$$
P_{\text {perc }}:=\inf _{k} \liminf _{N \rightarrow \infty} \mathbb{P}(0 \text { is linked by a path to a point at distance } k)>0,
$$

and $P_{\text {perc }}$ is then the probability of percolation. For each $k \geq 1$, let $\beta_{k} \in(0,1)$ satisfy

$$
\beta_{k}=1-\mathrm{e}^{-c_{k} \beta_{k-1}^{2} \beta_{k}}, \quad \beta_{0}=1,
$$

where $c_{k} \beta_{k-1}^{2}>1$.

Theorem 3. ([10].) Assume that $c_{k} \nearrow \infty$ as $k \rightarrow \infty, c_{1}>2 \log 2$, and $c_{2}>8 \log 2$. Then there is mean-field percolation if and only if $\sum_{k=1}^{\infty} \mathrm{e}^{-c_{k}}<\infty$, it occurs in the form of cascade percolation, and the probability of percolation is given by

$$
P_{\text {perc }}=\prod_{k=1}^{\infty} \beta_{k} .
$$

For example, with $c_{k}=a \log k$, there is mean-field percolation if and only if $a>1$. Cascade percolation means that the giant Erdös-Rényi components used in the proof at each level $k$ are made up of giant components at level $k-1$.

\subsection{Open problem}

In [11, Theorem 3.5] we considered the case of fixed $N, \delta=1, C_{2}=0$, and $c_{k_{n}}=$ $C+a N \log n$. We showed that percolation does not occur for $a<1$, while pre-percolation occurs for $a>1$, where, with $A_{n}$ denoting the annulus $B_{k_{n+1}}(0) \backslash B_{k_{n}}(0)$, pre-percolation is the situation where (with probability 1 ) there exists $n_{0}$ such that there is at least one edge connection from $A_{n}$ to $A_{n+1}$ for all $n \geq n_{0}$. To obtain percolation, in addition to pre-percolation, there would have to be paths connecting points in $A_{n+1}$ which are connected to $A_{n}$, to points in $A_{n+1}$ which are connected to $A_{n+2}$, for all $n \geq n_{0}$. That is, if $x$ and $y$ are points in $A_{n+1}$ such that 
$x$ is connected to $A_{n}$ and $y$ is connected to $A_{n+2}$, there would have to be a path connecting $x$ and $y$. An obvious question is whether there is percolation for some $a(N)$ which tends to 1 as $N \rightarrow \infty$. If not, in the $a>1$ case, does the expected size of the largest percolation cluster increase to $\infty$ as $N \rightarrow \infty$ ? If so, this would be consistent with the example for mean-field percolation in Subsection 3.3.

\section{Random walk on homogeneous ultrametric lattices}

\subsection{Hierarchical random walks}

A hierarchical random walk is a discrete-time walk on $\Omega_{\left\{N_{i}\right\}}$ that jumps distance $k \geq 1$ with probability $r_{k}\left(\sum_{k=1}^{\infty} r_{k}=1\right)$ and lands at a point chosen uniformly among the $\hat{N}_{k}-\hat{N}_{k-1}$ points at that distance from the previous position of the walk. In another model (not considered here) the walk jumps distance $k$ with probability $r_{k}$ and lands uniformly on the $k$-ball, rather than on the boundary of the ball as above. This other model is related to the mean-field analysis of interacting diffusions [13]. The two models are asymptotically equivalent as $N \rightarrow \infty$ because most of the points in a $k$-ball are at the boundary.

Flatto and Pitt [20] gave recurrence criteria for random walks on $\Omega_{\left\{N_{i}\right\}}$. In particular, if $\left\{N_{i}\right\}$ is bounded and $f_{n}=\sum_{j=n}^{\infty} r_{j}$, then the walk is recurrent if and only if $\sum_{n=1}^{\infty} 1 /\left(\hat{N}_{n-1} f_{n}\right)=\infty$. There are also results with unbounded $\left\{N_{i}\right\}$.

For $\Omega_{N}$, let $p_{t}(x, y)$ denote the transition probability of the continuous-time random walk (with unit-rate holding time). Using Fourier transforms, the following is obtained for $y$ at distance $k$ :

$$
\begin{gathered}
p_{t}(0, y)=\left(\delta_{0, k}-1\right) \frac{\mathrm{e}^{-h_{k} t}}{N^{k}}+(N-1) \sum_{j=k+1}^{\infty} \frac{\mathrm{e}^{-h_{j} t}}{N^{j}}, \quad t \geq 0, \\
h_{j}=\frac{N}{N-1} r_{j}+\sum_{i=j+1}^{\infty} r_{i}, \quad j=1,2, \ldots
\end{gathered}
$$

The special hierarchical random walk referred to in Subsection 2.2 is the $c$-random walk defined by

$$
r_{k}=\left(1-\frac{c}{N}\right)\left(\frac{c}{N}\right)^{k-1}, \quad k=1,2, \ldots,
$$

where $0<c<N$. In the next subsection we exhibit the asymptotic behaviour of the return probability $p_{t}(0,0)$ as $t \rightarrow \infty$ of this walk and see how it imitates a simple random walk on $\mathbb{Z}^{d}$. (See more on this in [16].)

\subsection{Degrees of transience and recurrence}

We introduced the concept of degree of transience and recurrence in [16]. If $X \equiv\{X(t), t \geq 0\}$ is a Lévy process on a Polish space $S$ (with additive Abelian group structure in our case), and $T_{t}$ is its semigroup, then the fractional powers of the potential operator $G$ of the process are defined on the space $B_{\mathrm{b}}(S)$ of bounded measurable functions with bounded support by

$$
G^{\zeta} \varphi=\frac{1}{\Gamma(\zeta)} \int_{0}^{\infty} t^{\zeta-1} T_{t} \varphi \mathrm{d} t \quad \zeta>0, \varphi \in B_{\mathrm{b}}(S) .
$$

The degree of $X$ is defined by

$$
\gamma=\sup \left\{\zeta>-1: G^{\gamma+1} \varphi<\infty \text { for all } \varphi \in B_{\mathrm{b}}^{+}(S)\right\} .
$$

Here $\gamma$ is the degree of transience if $\gamma>0$, and $-\gamma$ is the degree of recurrence if $-1<\gamma<0$. The $\gamma=0$ case is special because it may be that $|G \varphi|<\infty$ or $|G \varphi|=\infty$. 
For integers $k \geq 1, X$ is $k$-strongly transient if $\left\|G^{k+1} \varphi\right\|<\infty$, and $k$-weakly transient if $\left\|G^{k} \varphi\right\|<\infty$ and $G^{k+1} \varphi \equiv \infty$. The $k=1$ case corresponds to the usual strong/weak transience. A basic role in occupation time fluctuation limits of multilevel branching particle systems is played by $k$-strong/weak transience, as we note in Subsection 5.2.

The degree of transience is also given by

$$
\gamma=\sup \left\{\zeta \geq 0: \mathbb{E}\left[L_{B_{R}}^{\zeta}\right]<\infty \text { for all } R>0\right\},
$$

where $L_{B_{R}}$ is the last exit time from a ball of radius $R$ centered at the initial point of the walk. For an irreducible transient random walk on a countable Abelian group, $L_{B_{R}}$ may be replaced by the last exit time from the initial point.

For an $\alpha$-stable (symmetric Lévy) process on $\mathbb{R}^{d}$, or corresponding random walk on $\mathbb{Z}^{d}$, $0<\alpha \leq 2$, the degree is

$$
\gamma=\frac{d}{\alpha}-1
$$

and for $c$-random walk on $\Omega_{N}$, the degree is

$$
\gamma=\frac{\log c}{\log (N / c)} .
$$

In the Euclidean case $\gamma$ can only take values in $\left[-\frac{1}{2}, \infty\right)$, whereas in the ultrametric case $\gamma$ can take any value in $(-1, \infty)$. In this sense the $c$-random walks form a richer class of processes. Transience/recurrence properties of $\alpha$-stable processes and $c$-random walks are given in Subsection 5.2.

As $t \rightarrow \infty$, for the $\alpha$-stable process,

$$
p_{t}(0,0) \sim(\text { const. }) t^{-(\gamma+1)}=(\text { const. }) t^{-d / \alpha},
$$

while, for the $c$-random walk,

$$
\left.p_{t}(0,0) \sim(\text { const. }) t^{-(\gamma+1)} h_{t}=\text { (const. }\right) t^{-\log N / \log (N / c)} h_{t},
$$

where $h_{t}$ is a function which oscillates in logarithmic scale between two positive values. Thus, the long-time behaviours of the two processes are analogous for the same values of $\gamma$. In addition, $G \varphi \equiv \infty$ in both cases. Hence, there is analogy at the border between recurrence and transience for a simple random walk on $\mathbb{Z}^{2}(\gamma=0, c=1, d=2)$, called critical recurrence. This also holds for Brownian motion on $\mathbb{R}^{2}$, but there are no nontrivial continuous processes on $\Omega_{N}$. Note also that, for the $c$-random walk, $\gamma \rightarrow 0$ as $N \rightarrow \infty$. Hence, the mean-field limit of the $c$-random walk corresponds to Brownian motion or a simple random walk in dimension 2, approaching from the transient side if $c>1$ and from the recurrent side if $c \leq 1$. (See more on this in [17].)

For $\gamma>0$, the potential kernel of the $c$-random walk is $G(x)=N^{-|x| \gamma /(\gamma+1)}$. Equating the $\gamma$ s for the $\alpha$-stable process and $c$-random walk gives $G(x)=$ (const.) $\rho(x)^{-(d-\alpha)}$, where $\rho(x)=N^{|x| / d}$ is the 'Euclidean radial distance' from 0 to $x$, so that the volume of a ball of radius $\rho$ grows like $\rho^{d}$ (note that $N^{|x|}$ is also an ultrametric on $\Omega_{N}$ ).

It follows from these observations that the $\alpha$-stable process on $\mathbb{R}^{d}$ (and corresponding random walk on $\mathbb{Z}^{d}$ ) and the $c$-random walk on $\Omega_{N}$ with $c=N^{1-\alpha / d}$ have analogous behaviours. The analogy is relevant in [22]. The analogy also allows us to choose the $c$-random walk which is a caricature of what would be the random walk on $\mathbb{Z}^{d}$ with noninteger dimension $d$ (e.g. $d=4-\varepsilon$ in some problems in statistical physics [7]). There are also differences due to 
ultrametricity (e.g. the distance from the origin behaves differently for the Euclidean random walk and $c$-random walk; see [16, Remark 3.5.7]). More on degrees and their role is given in [14] and [16]. The results are used in [15] to study hierarchical equilibria of branching systems.

\subsection{Nearest-neighbour random walks on percolation clusters}

We consider a simple random walk on the percolation cluster in $\Omega_{N}$. Due to the lack of group structure of the percolation cluster, the usual criteria for transience/recurrence cannot be applied. However, methods based on the relation with electrical circuits can be used [31], [32]. These methods have been used for random walks on percolation clusters in Euclidean lattices for Bernoulli and long-range percolation [23], [2], and were extended to the ultrametric setting in [12].

A unit flow on an infinite graph $g=(V, \mathscr{E})$ with source $a \in V$ is a function $\theta$ on the set of edges $\&$ such that $\theta(x, y)=-\theta(y, x)$,

$$
\sum_{x \neq a} \theta(a, x)=1 \quad \text { and } \quad \sum_{y \sim x} \theta(x, y)=0 \quad \text { for all } x \neq a,
$$

where now $x \sim y$ means that $y$ is a neighbour of $x$. The energy of the flow is

$$
\tilde{E}(\theta)=\sum_{e \in \mathcal{E}^{*}}(\theta(e))^{2} R(e)
$$

where $\varepsilon^{*}$ is the set of directed edges and $R(e):=R(x, y)$ is the resistance of the edge $e$ from $x$ to $y$ (in the present context the resistance of an edge is 1). A random walk on a countable connected graph $g$ is transient if and only if the resistance from a designated vertex to infinity is finite. In turn this is equivalent to the existence of a unit flow from any vertex $a$ to $\infty$ on $g$ with finite energy [31].

Consider the percolation cluster in the $\delta<1$ case in Theorem 1 above.

Theorem 4. ([12].) Consider $\Omega_{N}$ in the $\delta<1$ case. Then, for almost every realization of the percolation cluster, the random walk on the cluster is transient.

Proof. We outline the argument of [12, Theorem 4.4] and indicate how it can be extended to the more general case of percolation on $\Omega_{\left\{N_{i}\right\}}$. In terms of the properties of the clusters constructed in Theorem 1, the idea is to embed a tree structure in the infinite cluster with root at 0 and, using branches passing through the sequence of annuli $A_{n}=B_{k_{n+1}}(0) \backslash B_{k_{n}}(0)$, to verify that the resistance along the tree from 0 to $\infty$ is finite.

Using $\tilde{q}_{n}$ as in the proof of Theorem 1 , we can show that, for large $n$, we can pick $4^{n}$ direct edges from $A_{n-1}$ to $A_{n}$. Then, using results on path lengths in the infinite cluster as in [12], we can connect the resulting entrance and exit vertices in the $A_{n}$ by paths of length smaller than $3^{n}$. The proof now follows since the tree from the root to $\infty$ can be viewed as a series of $4^{n}$ circuits in parallel connecting the set of entrance vertices of $A_{n-1}$ and the set of entrance vertices of $A_{n}$ with connecting circuits within the individual annuli having resistance less than $3^{n}$. Therefore, the resistance of the tree from the root to $\infty$ is

$$
C \sum_{n=n_{0}}^{\infty} \frac{3^{n}}{4^{n}}<\infty
$$

for some constant $C$. A careful inspection of the proof given in [12] shows that the relevant calculations are applicable to percolation on $\Omega_{\left\{N_{i}\right\}}$ provided that $N_{i} \geq 2$ for all $i$. 
The critical case, $\delta=1$, is of special interest because both transience and recurrence can occur. Recall that percolation occurs in this case with connection probabilities as in Theorem 2 with $C_{2}>0$ and $\alpha>0$.

Theorem 5. ([12].) For $\Omega_{N}$, consider the simple random walk on the percolation cluster with $\delta=1$ and $C_{2}>0$. Then, for almost every realization of the percolation cluster, there exists a critical $\alpha_{\mathrm{c}} \in(0, \infty)$ such that, for $\alpha<\alpha_{\mathrm{c}}$, the random walk is recurrent and, for $\alpha>\alpha_{\mathrm{c}}$, the random walk is transient.

\subsection{Open problems}

An open problem concerning Theorem 5 is to determine the value of $\alpha_{\mathrm{c}}$, and whether the walk is transient or recurrent at $\alpha_{\mathrm{c}}$.

Comparisons between random walks on long-range percolation clusters in $\mathbb{Z}^{d}[2]$ and in $\Omega_{N}$ are made in [12]. The determination of the degrees of transience and recurrence of the random walk on the percolation cluster of $\mathbb{Z}^{d}$ and $\Omega_{N}$ is an open problem.

The spectral dimension of a transient simple random walk on an infinite graph with $n$-step transition probability $p_{n}(x, y)$ is defined by

$$
d_{s}=-2 \lim _{n \rightarrow \infty} \frac{p_{2 n}(x, x)}{\log n}
$$

if the limit exists (see, e.g. [27]). Using the criterion for finiteness of moments of the last exit time from a finite set in [38], it is easy to see that the degree of transience and the spectral dimension are related by

$$
\gamma=\frac{1}{2} d_{s}-1
$$

Heat kernel bounds and scaling limits of the random walk on the long-range percolation clusters in $\mathbb{Z}^{d}$ are determined in [8] and [9]. It is an open problem to obtain analogous results for $\Omega_{N}$.

\section{Interacting particle systems}

\subsection{Universality classes of long-time behaviour}

For many spatially interacting systems involving random walks, there are universality classes of long-time behaviour that depend only on the degree of transience and recurrence of the random walk, for example, the behaviour is the same in the Euclidean and ultrametric cases having the same degree, as we have seen in Subsection 4.2. Examples include branching particle systems, voter models, and depinning [39]. The case of occupation time fluctuations for branching systems was studied in a series of papers [14], [4], [5], [6]. These results are reviewed in the next subsection.

On the other hand, there are some significant differences between the Euclidean and ultrametric geometries. In particular, the scaling limits in the two cases differ as follows. In the ultrametric case there is no scaling limit with continuous paths and, therefore, no Brownian motion. However, there exist stable Lévy processes in the continuum limit of random walks on the ultrametric hierarchical lattices $\Omega_{N}^{L}$ (with lattice spacing $\mathrm{e}^{-L}$ ) for any stable index in $(0, \infty)$ [17], [19], but there is no stable process with index in $(2, \infty)$, that is, degree in $\left(-1,-\frac{1}{2}\right)$ in the Euclidean case.

\subsection{Occupation time fluctuations of branching particle systems}

In [14] we studied occupation time fluctuation limits as time $t \rightarrow \infty$ of particle systems on $\mathbb{R}^{d}$ and on $\Omega_{N}$ called $k$-level systems, $k=0,1,2$; these correspond respectively to no branching, individual particle branching, and, in addition to individual branching, branching 
of families of related particles (called 2-level branching). The branching laws have mean 0 and finite variance. In $\mathbb{R}^{d}$ the particles perform $\alpha$-stable process, and in $\Omega_{N}$ they perform a $c$-random walk. Descriptions of the systems and the results are too long to be included here. The point is that, for both models, Euclidean and ultrametric, the results are analogous and depend only on the degree of transience/recurrence $\gamma$. Recall that, for the $\alpha$-stable process, $\gamma=d / \alpha-1$ and, for the $c$-random walk, $\gamma=\log c / \log (N / c)$, and that, for the same values of $\gamma$, both processes have analogous long-time behaviours.

The normings for the occupation time fluctuations at time $t$ and the nature of the limits as $t \rightarrow \infty$ (Gaussian random fields on $\mathbb{R}^{d}$ and $\Omega_{N}$ ) are different according to transience/recurrence properties of the particle process, as follows (see Subsection 4.2 for $k$-weak/strong transience).

0-level: $\bullet-1<\gamma<0(d<\alpha, c<1$, recurrence $)$,

- $\gamma=0(d=\alpha, c=1$, recurrence at border to transience $)$,

- $\gamma>0(d>\alpha, c>1$, transience $)$.

1-level: $\bullet 0<\gamma<1\left(\alpha<d<2 \alpha, 1<c<N^{1 / 2}\right.$, weak transience),

- $\gamma=1\left(d=2 \alpha, c=N^{1 / 2}\right.$, weak transience at border to strong transience),

- $\gamma>1\left(d>2 \alpha, c>N^{1 / 2}\right.$, strong transience $)$.

2-level: $\bullet 1<\gamma<2\left(2 \alpha<d<3 \alpha, N^{1 / 2}<c<N^{2 / 3}\right.$, 2-weak transience),

- $\gamma=2\left(d=3 \alpha, c=N^{2 / 3}, 2\right.$-weak transience at border to 2-strong transience),

- $\gamma>2\left(d>3 \alpha, c>N^{2 / 3}, 2\right.$-strong transience $)$.

For each $k$-level system, there are three cases: small $\gamma$, border $\gamma$, and large $\gamma$. For small $\gamma$, the order of the occupation time fluctuations, which determine the norming, is $t^{\delta / 2}$ for some $\delta>1(\delta=1-\gamma$ for $-1<\gamma<0, \delta=2-\gamma$ for $0<\gamma<1$, and $\delta=3-\gamma$ for $1<\gamma<2)$; for border $\gamma$, the order is $(t \log t)^{1 / 2}$; for large $\gamma$, it is $t^{1 / 2}$.

Interpretations of the results can be given in terms of transience/recurrence properties of the particle process. For $k=2$, the 'particles' correspond to clans of family-related individual particles. For the 2-level system, owing to the strong transience of the individual particle process, the system (with a suitable initial condition) converges in distribution as $t \rightarrow \infty$ towards a nontrivial equilibrium state (see [15, Proposition 2.3.5] for the hierarchical model).

If the time is rescaled by $T$ and $T \rightarrow \infty$, there are functional central limit theorems for the occupation time fluctuation process in appropriate spaces for $k=0,1$ [4], [5], [6]. We mention the temporal parts of the limit processes for small $\gamma$ because of the long-range dependence properties they have.

For the Euclidean model, the limit for the 0-level system with $\gamma<0$ (recurrence) is a long-range dependent Gaussian process called fractional Brownian motion, with covariance function of the form

$$
s^{2 H}+t^{2 H}-|s-t|^{2 H}, \quad s, t \geq 0,
$$

$H=1-1 / 2 \alpha \in\left(\frac{1}{2}, \frac{3}{4}\right], \alpha \in(1,2], d=1$, and the limit for the 1-level system with $0<\gamma<1$ (weak transience) is a long-range dependent Gaussian process called sub-fractional Brownian motion, with covariance function of the form

$$
\begin{gathered}
s^{2 H}+t^{2 H}-\frac{1}{2}\left[(s+t)^{2 H}+|s-t|^{2 H}\right], \quad s, t \geq 0, \\
H=\frac{1}{2}(3-d / \alpha) \in\left(\frac{1}{2}, 1\right), \alpha \in\left(\frac{3}{4}, 1\right], \alpha<d<2 \alpha .
\end{gathered}
$$


For the border and large $\gamma$, the limit processes are also Gaussian, but there are no long-range dependent effects because the increments of the processes are independent.

Analogous limit processes for the ultrametric models with $\gamma<0$ and $0<\gamma<1$ are long-range dependent Gaussian processes with covariance functions of the forms

$$
\begin{gathered}
s^{2 H} h_{s}+t^{2 H} h_{t}-|s-t|^{2 H} h_{|s-t|}, \quad s, t \geq 0, \\
s^{2 H} h_{s}^{\prime}+t^{2 H} h_{t}^{\prime}-\frac{1}{2}\left[(s+t)^{2 H} h_{s+t}^{\prime}+|s-t|^{H} h_{|s-t|}^{\prime}\right], \quad s, t \geq 0,
\end{gathered}
$$

where the functions $h_{t}$ and $h_{t}^{\prime}$ oscillate in logarithmic scale between two positive values. These processes are ultrametric analogues of fractional and sub-fractional Brownian motions.

\subsection{Open problem}

Functional central limit theorems should also exist for the Euclidean and ultrametric 2-level branching systems, with long-range dependence in the $1<\gamma<2$ case (2-weak transience), as well as with some infinite variance branchings at one or both of the branching levels.

\subsection{Particle systems on percolation clusters}

Percolation clusters can serve as spatial environments for population models. The random nature of the percolation cluster can reflect the local irregularity of real-world environments. For example, contact and voter processes on infinite percolation clusters in $\mathbb{Z}^{d}$ as models of host-symbiont interactions are studied in [3]. However, since the random walk on the cluster is not symmetric, the group property cannot be used and the analysis is more difficult. The coalescing random walk serves as the dual for the voter model [29, Chapter V] and the long-time behaviour of the voter model (started with i.i.d. conditions) depends on whether the random walk has the finite (coexistence) or infinite meeting property (locally monotype). The meeting property also plays a role for critical branching systems. For example, Proposition 4.2 of [30] states that the infinite meeting property implies the local extinction of a critical branching system with uniform intensity.

A random walk on a group with $p(x, y)=p(0, y-x)$ has the infinite meeting property if and only if it is recurrent [37, Section 4]. Therefore, the infinite meeting property is satisfied for the hierarchical random walks in Subsections 4.1 and 4.2 in the case of recurrence ( $c \leq 1$ for the $c$-random walk on $\Omega_{N}$ ). However, in the more general case there exist recurrent symmetric Markov chains on graphs for which, with probability 1, the number of meetings is finite [26], [28].

\subsection{Open problem}

It is an open problem to determine whether there is positive probability of infinitely many meetings in the recurrent case in Theorem 5. The infinite meeting property is established for the infinite percolation cluster for Bernoulli percolation in $\mathbb{Z}^{2}$ with $p>p_{c}$ in [3].

In [26] it is noted that if the degree of the graph is bounded then in the transient case the expected number of meetings is finite. We conjecture that this finiteness is also true in the transient case in Theorem 5 even though the degree is unbounded.

For the voter model, coexistence occurs in the transient case (with bounded degree) since there is only a finite number of meetings and, therefore, there is positive probability that coalescence does not occur.

\section{Acknowledgements}

We thank Anton Wakolbinger and the referee for comments that helped us to improve the presentation. 


\section{References}

[1] Avetisov, V. A., Bikulov, A. K. And Zubarev, A. P. (2014). Ultrametric random walk and dynamics of protein molecules. Proc. Steklov Inst. Math. 285, 3-25.

[2] Berger, N. (2002). Transience, recurrence and critical behavior for long-range percolation. Commun. Math. Phys. 226, 531-558.

[3] Bertacchi, D., Lanchier, N. And Zucca, F. (2011). Contact and voter processes on the infinite percolation cluster as models of host-symbiont interactions. Ann. Appl. Prob. 21, 1215-1252.

[4] Bojdecki, T., Gorostiza, L. G. and TalarczyK, A. (2006). Limit theorems for occupation time fluctuations of branching systems. I. Long-range dependence. Stoch. Process. Appl. 116, 1-18.

[5] Bojdecki, T., Gorostiza, L. G. and TalarczyK, A. (2006). Limit theorems for occupation time fluctuations of branching systems. II. Critical and large dimensions. Stoch. Process. Appl. 116, 19-35.

[6] Bojdecki, T., Gorostiza, L. G. and TalarczyK, A. (2013). Oscillatory fractional Brownian motion. Acta Appl. Math. 127, 193-215.

[7] Collet, P. and Eckmann, J.-P. (1978). A Renormalization Group Analysis of the Hierarchical Model in Statistical Mechanics (Lecture Notes Phys. 74). Springer, Berlin.

[8] Crawford, N. AND SLY, A. (2012). Simple random walk on long range percolation clusters I: heat kernel bounds. Prob. Theory Relat. Fields 154, 753-786.

[9] Crawford, N. ANd SLY, A. (2013). Simple random walk on long-range percolation clusters II: scaling limits. Ann. Prob. 41, 445-502.

[10] Dawson, D. A. and Gorostiza, L. G. (2007). Percolation in a hierarchical random graph. Commun. Stoch. Anal. 1, 29-47.

[11] Dawson, D. A. and Gorostiza, L. G. (2013). Percolation in an ultrametric space. Electron. J. Prob. 18, 26pp.

[12] Dawson, D. A. And Gorostiza, L. G. (2018). Transience and recurrence of random walks on percolation clusters in an ultrametric space. J. Theoret. Prob. 31, 494-526.

[13] Dawson, D. A. And Greven, A. (1993). Hierarchical models of interacting diffusions: multiple time scale phenomena, phase transition and pattern of cluster-formation. Prob. Theory Relat. Fields 96, 435-473.

[14] Dawson, D. A., Gorostiza, L. G. and Wakolbinger, A. (2001). Occupation time fluctuations in branching systems. J. Theoret. Prob. 14, 729-796.

[15] Dawson, D. A., Gorostiza, L. G. and Wakolbinger, A. (2004). Hierarchical equilibria of branching populations. Electron. J. Prob. 9, 316-381.

[16] Dawson, D. A., Gorostiza, L. G. And Wakolbinger, A. (2005). Degrees of transience and recurrence and hierarchical random walks. Potential Anal. 22, 305-350.

[17] Dawson, D. A., Greven, A. And Z̈̈hle, I. (2018). Continuous limits of multitype population models on the hierarchical group. In preparation.

[18] Dragovich, B., Khrennikov, A. Y., Kozyrev, S. V. and Volovich, I. V. (2009). On p-adic mathematical physics. p-Adic Numbers Ultrametric Anal. Appl. 1, 1-17.

[19] Evans, S. N. (1989). Local properties of Lévy processes on a totally disconnected group. J. Theoret. Prob. 2, 209-259.

[20] Flatto, L. ANd Pitt, J. (1974). Recurrence criteria for random walks on countable Abelian groups. Illinois J. Math. 18, 1-19.

[21] Greven, A., Pfaffelhuber, P. And Winter, A. (2013). Tree-valued resampling dynamics martingale problems and applications. Prob. Theory Relat. Fields 155, 789-838.

[22] Greven, A., Den Hollander, F., Kliem, S. and Klimovsky, A. (2014). Renormalisation of hierarchically interacting Cannings processes. ALEA 11, 43-140.

[23] Grimmett, G. R., Kesten, H. and Zhang, Y. (1993). Random walk on the infinite cluster of the percolation model. Prob. Theory Relat. Fields 96, 33-44.

[24] Janson, S., ŁuczaK, T. And Rucinski, A. (2000). Random Graphs. Wiley-Interscience, New York.

[25] Koval, V., Meester, R. and Trapman, P. (2012). Long-range percolation on the hierarchical lattice. Electron. J. Prob. 17, 21pp.

[26] Krishnapur, M. AND Peres, Y. (2004). Recurrent graphs where two independent random walks collide finitely often. Electron. Commun. Prob. 9, 72-81.

[27] KumagaI, T. (2014). Random Walks on Disordered Media and Their Scaling Limits (Lecture Notes Math. 2101). Springer, Cham.

[28] Liggett, T. M. (1974). A characterization of the invariant measures for an infinite particle system with interactions. II. Trans. Amer. Math. Soc. 198, 201-213.

[29] Liggett, T. M. (1985). Interacting Particle Systems (Fundamental Principles Math. Sci. 276). Springer, New York.

[30] López-Mimbela, J. A. And WaKolbinger, A. (1997). Which critically branching populations persist? In Classical and Modern Branching Processes (IMA Vol. Mat. Appl. 84), eds K. B. Athreya and P. Jagers, Springer, New York, pp. 203-216. 
[31] Lyons, T. (1983). A simple criterion for transience of a reversible Markov chain. Ann. Prob. 11, $393-402$.

[32] Lyons, R. and Peres, Y. (2016). Probability on Trees and Networks. Cambridge University Press.

[33] Panchenko, D. (2013). The Parisi ultrametricity conjecture. Ann. Math. 177, 383-393.

[34] Rammal, R., Toulouse, G. and Virasoro, M. A. (1986). Ultrametricity for physicists. Rev. Modern Phys. 58, $765-788$.

[35] Schiкhof, W. H. (1984). Ultrametric Calculus: An Introduction to p-Adic Analysis. Cambridge University Press.

[36] Shang, Y. (2012). Percolation in a hierarchical lattice. Z. Naturforsch. 67, 225-229.

[37] Spitzer, F. (1974). Recurrent random walk of an infinite particle system. Trans. Amer. Math. Soc. 198, $191-199$.

[38] Takeuchi, J. (1967). Moments of the last exit times. Proc. Japan Acad. 43, 355-360.

[39] VandembroucQ, D. and Roux, S. (2004). Large-scale numerical simulations of ultrametric long-range depinning. Phys. Rev. E 70, 026103, 9pp.

\section{A. DAWSON, Carleton University}

School of Mathematics and Statistics, Carleton University, Ottawa, Ontario K1S 5B6, Canada.

\section{G. GOROSTIZA, CINVESTAV}

Department of Mathematics, CINVESTAV-IPN, Apartado Postal 14-740, Mexico DF 07000, Mexico.

Email address: 1gorosti@math.cinvestav.mx 